\title{
KIF3A and IL-4 are disease-specific biomarkers for psoriatic arthritis susceptibility
}

\author{
Raffaella Cascella ${ }^{1,2, *}$, Claudia Strafella ${ }^{3,4, *}$, Michele Ragazzo ${ }^{1,5}$, Laura Manzo ${ }^{3,4}$, \\ Gaetana Costanza ${ }^{6}$, John Bowes7, Ulrike Hüffmeier ${ }^{8}$, Saverio Potenza ${ }^{3}$, Federica \\ Sangiuolo ${ }^{3}$, André Reis ${ }^{8}$, Anne Barton ${ }^{7,9}$, Giuseppe Novelli ${ }^{3}$, Augusto Orlandi ${ }^{10}$ and \\ Emiliano Giardina ${ }^{1,3}$ \\ ${ }^{1}$ Molecular Genetics Laboratory UILDM, Santa Lucia Foundation, Rome, Italy \\ ${ }^{2}$ Department of Chemical Pharmaceutical and Biomolecular Technologies, Catholic University "Our Lady of Good Counsel" \\ Laprakë, Rruga Dritan Hoxha, Tirana, Albania \\ ${ }^{3}$ Department of Biomedicine and Prevention, "Tor Vergata" University, Rome, Italy \\ ${ }^{4}$ Emotest Laboratory, Pozzuoli, Italy \\ ${ }^{5}$ Department of Medical Science, Catholic University "Our Lady of Good Counsel" Laprakë, Rruga Dritan Hoxha, Tirana, \\ Albania \\ ${ }^{6}$ Anatomic Pathology, Department of Biomedicine and Prevention, Tor Vergata University of Rome, Rome, Italy \\ ${ }^{7}$ Arthritis Research UK Centre for Genetics and Genomics, The University of Manchester, Manchester, UK \\ ${ }^{8}$ Institute of Human Genetics, University of Erlangen-Nuremberg, Erlangen, Germany \\ ${ }^{9}$ NIHR Manchester Musculoskeletal Biomedical Research Unit, Central Manchester Foundation Trust and University of \\ Manchester, Manchester Academy of Health Sciences, Manchester, UK \\ ${ }^{10}$ Anatomic Pathology, Department of Biomedicine and Prevention, Tor Vergata University of Rome, Italy, Tor Vergata \\ University Hospital, Rome, Italy \\ *These authors have contributed equally to this work \\ Correspondence to: Raffaella Cascella, email: raffaellacascella@virgilio.it \\ Keywords: psoriatic arthritis; susceptibility; bone metabolism; 5 q3 1 locus; linkage disequilibrium \\ Received: June 22, $2017 \quad$ Accepted: August 06, $2017 \quad$ Published: September 08, 2017 \\ Copyright: Cascella et al. This is an open-access article distributed under the terms of the Creative Commons Attribution License \\ 3.0 (CC BY 3.0), which permits unrestricted use, distribution, and reproduction in any medium, provided the original author and \\ source are credited.
}

\section{ABSTRACT}

To date, the genes associated with Psoriatic Arthritis (PsA) are principally involved in inflammation, immune response and epidermal differentiation, without any information about the relationship between disease and bone metabolism genes. Our work was focused on 5q31 locus, which contains several genetic variants significantly associated with PsA. The study involved 1526 subjects (500 PsA, 426 PsV, 600 controls). The region was evaluated by selecting and genotyping the SNPs of interest by Real Time PCR and direct sequencing. The results were subjected to biostatistic and bioinformatic analysis.

The case-control study highlighted a significant association between KIF3A/IL4 and PsA, but not with PsV (Psoriasis Vulgaris) patients. In addition, the haplotype analysis revealed two haplotypes significantly associated with PsA susceptibility. The Linkage Disequilibrium (LD) study showed the presence of a specific block in high LD within $132,692,628-132,737,638$ bp of 5q31, giving additional evidence of specific association of the 5q31 region in PsA patients. Moreover, KIF3A expression was assessed by immunohistochemistry assays which showed a marked and significant difference of KIF3A expression between pathological and normal tissues. Our analysis described KIF3A and IL-4 as novel susceptibility genes for PsA, suggesting a clear implication of bone metabolism genes in the disease etiopathogenesis. 


\section{INTRODUCTION}

Extensive research has been carried out in order to draw an exhaustive genomic picture of the etiopathology of Psoriatic Arthritis (PsA, OMIM \#607507). Up to date, most of the knowledge concerning the etiopathogenetic mechanisms of PsA essentially refers to genes involved in inflammation, immune response and epidermal differentiation [1-3]. PsA is an inflammatory arthropathy caused by the erosion and inflammation of the distal joints (in particular the interphalangeal and sacroiliac joints) and the entheses (the insertion sites between the tendon and the bone) with a possible involvement of the axial skeleton. The disease can also be characterized by dysregulated bone formation at the level of the peripheral or axial skeleton. The degree of severity and the course of PsA are variable, ranging from mild to more severe forms, leading to a destructive and progressively debilitating course [4]. The prevalence of PsA is estimated to be $0.3 \%-1 \%$ in the general population, although it is often developed in combination with Psoriasis Vulgaris (PsV, OMIM $\# 177900$; in $20-30 \%$ of cases), and it presents an incidence peak between 20 and 40 years of age independent of gender [5-7].

Even though the pathogenetic molecular pathways are still unclear, the damage in PsA is known to be correlated to inflammatory events that enhance the production of inflammatory molecules and the activation of immune cells (T-cells). The migration of inflammatory molecules and T-cells to the synovia thereby disrupts the metabolic and remodelling (osteoblasts/osteoclasts differentiation) activities of the bone. This process finally results in the erosion, osteolysis and loss of Bone Mineral Density (BMD), which are responsible of the bone deformation and destruction in PsA [3]. Consequently, common symptoms of the disease are inflammation of the joints which become painful, stiff, swollen and hot.

PsA is classified as a multifactorial disease in which environmental and genetic factors are likely to influence susceptibility to the disease. Environmental factors include stress, low-humidity, drugs, smoking, obesity and chronic infections [7]. The genetic contribution has been extensively investigated through a number of studies (linkage analysis on twins and families, case-control studies, and genome-wide association studies). In fact, a recurrence risk of 40 has been reported among first-degree relatives of patients with PsA $[1,7]$. Several susceptibility genes have been found to confer a higher risk for PsA (and PsV for most of them), such as HLA $\left(C w^{*} 06: 02, B^{*} 08, B^{*} 27\right.$, $\left.B^{*} 38\right), L C E 3 B, L C E 3 C, T R A F 3 I P 2, I L s$ (IL-17, IL-23, IL23R, IL12, IL12B, IL21), RUNX3, TNF- $\alpha$, TNIP1, PTPN22, $D E N N D 1 B, S L C 22 A 5$ [8-15]. As expected, all of the genes are involved in inflammatory, immunologic and epidermal differentiating mechanisms. However, information about the genes potentially implicated in the disruption of bone homoeostasis in PsA has yet to be clarified.
In this context, the investigation of such genes may reveal attractive insights into the principal mechanisms of the disease. Given these premises, we focused our attention on the $5 \mathrm{q} 31$ locus, which contains several genetic variants significantly associated with PsA $[14,16]$ as well as a number of genes (280, including $A D R B 2, S P A R C$, TGFBI, IL-4, KIF3A, SMAD5, GDF9, PDLIM4) involved in the osteogenic, differentiating and remodelling activities of the bone. Among them, KIF3A (Kinesin Family Member $3 A$ ) caught our attention as it was previously associated with other multifactorial disorders that share etiopathogenetic pathways similar to PsA. In fact, KIF3A has been associated with Atopic Eczema (AE), which is a chronic inflammatory skin disease characterized by a multifactorial inheritance pattern [17]. Genetic evidence highlighted the implication of genes involved in skin barrier dysfunction, excessive immune and inflammatory responses which are similar in PsA. KIF3A has a size of 47,986 bases and includes 17 exons and 20 introns. $K I F 3 A$ codes for the homonymous protein (KIF3A) that is a member of the Kinesin family protein (a class of motor proteins involved in the intracellular transport). KIF3A is ubiquitously expressed in several tissues, such as nervous tissue, bone, skin, kidney. The protein takes part in several molecular pathways including skin homoeostasis and skeletal morphogenesis. Studies on KIF3A knockout mice demonstrated that the gene ablation led to disruption of the normal skeletal development [18-20]. Given these data, KIF3A represents a good positional and functional candidate gene potentially involved in PsA etiopathogenesis. Thus, we decided to investigate the KIF3A gene and its flanking regions $(132,692,628$ $132,737,638 \mathrm{bp}$ ) as a susceptibility locus in PsA.

\section{RESULTS}

\section{Genetic studies}

Our research work essentially explored $5 \mathrm{q} 31$ locus, with a particular focus on $K I F 3 A$ gene and its flanking regions $(132,692,628-132,737,638 \mathrm{bp})$. To this purpose, we performed an in-silico study of SNPs able to describe the maximum variability of the $5 \mathrm{q} 31$ genomic region of interest, paying attention to the frequency and the specific position of variants throughout the locus. The analysis led to the selection of four SNPs that are rs2227282 (C/G), rs2285700 (A/C), rs10062446 (A/T), and rs2897442 $(\mathrm{A} / \mathrm{G})$. However, a careful bioinformatic research indicated that rs 2227282 was localized in the $I L-4$ gene, while the rs2285700, rs10062446, and rs2897442 were positioned in the KIF3A gene (Table 1, Figure 1).

The case-control study (1526 subjects, including 500 PsA, 426 PsV, 600 controls) revealed significant allele association with susceptibility to PsA (Table 2). In fact, the rs2227282 had a $p=2.09601^{*} 10^{-5}$ and OR $(\mathrm{G})=1.4$ (95\%CI: $1.12-1.73$ ). The rs2285700 had a $p=3^{*} 10^{-3}$ and 
Table 1: The selected SNPs on genomic regions falling within 132,692,628-132,737,638 bp (5q31). ( $)$ : db SNP on NCBI Reference Assembly; (\#): HapMap refSNP

\begin{tabular}{lcccc}
\hline SNPs & Position $(*)$ & Gene $\left(^{*}\right)$ & Alleles $\left(^{*}\right)$ & Frequencies $\left(^{*}\right)$ \\
\hline rs2227282 & 132.677 .487 & $I L-4$ & C/G & G: $0.735 ;$ C: 0.265 \\
rs2285700 & 132.703 .440 & $K I F 3 A$ & A/C & A: $0.726 ;$ C: 0.274 \\
rs10062446 & 132.704 .682 & $K I F 3 A$ & A/T & A: $0.721 ;$ T: 0.279 \\
rs2897442 & 132.713 .335 & $K I F 3 A$ & A/G & A: $0.692 ;$ G: 0.308 \\
\hline
\end{tabular}

OR $(\mathrm{A})=1.3(95 \% \mathrm{CI}: 1.01-1.60)$. The rs $10062446 \mathrm{had}$ a $p=5^{*} 10^{-3}$ and OR $(\mathrm{A})=1.3(95 \% \mathrm{CI}: 1.0-1.57)$. The rs 2897442 had $p=1.2^{*} 10^{-4}$ and OR $(\mathrm{A})=1.4(95 \% \mathrm{CI}: 1.09$ 1.71). Interestingly, the same SNPs were further genotyped in the cohort of PsV patients ( $\mathrm{n}=426)$ without reporting a significant association. This result indicates that PsA and $\mathrm{PsV}$ are characterized by genetic signatures which confer specific susceptibility to develop one disease or another. Given these data, $I L-4$ and $K I F 3 A$ are genetically associated exclusively with PsA. The SNPs of interest are mapped within the intronic regions of the genes, and the prediction analysis for the impact of these SNPs did not reveal substantial alteration of the splice sites. This result suggested that the SNPs may have an impact on the proteins other than a change of splicing activity. Given the association of $I L-4$ and KIF3A with PsA and their closeness within the $5 \mathrm{q} 31$ locus, we evaluated the Linkage Disequilibrium (LD) pattern in cases and control samples. The LD analysis was performed in order to further support our results and provide additional evidence of specific association of $5 \mathrm{q} 31$ with PsA. As expected, the LD patterns between the two groups were almost overlapping $\left(\mathrm{D}^{\prime}=0.95\right)$ revealing two PsA-associated haplotypes, C-A-A-A with $p=1.70652^{*} 10^{-6}$ and $\mathrm{OR}=1.6$ (95\%CI: $1.2-2.0)$ and G-C-T-G with $p=1.70652^{*} 10$ ${ }^{6}$ and $\mathrm{OR}=0.6$ (95\%CI: $\left.0.5-0.8\right)$, covering up to $97 \%$ of the variability of $132,692,628-132,737,638 \mathrm{bp}$ of the $5 \mathrm{q} 31$ locus. As expected, the risk haplotype (C-A-A-A) reported a higher frequency in cases than in control subjects, while the protective haplotype (G-C-T-G) was more frequent in control samples (Table 3). Successively, a wider investigation of $K I F 3 A$ locus revealed two SNPs, rs2277065 (A/G) and rs2277066 (G/C), within the promoter region that were significantly associated with PsA. In particular, the rs2277065 reported a $p=0.001$ and OR $(A)=1.70(95 \%$ CI: $1.22-2.36)$ and the rs 2277066 had a $p=0.004$ and $\mathrm{OR}=1.52$ (95\%CI: 1.14-2.04) (Table 4). In addition, we assessed the potential impact of the SNPs on the splicing activity through the bioinformatic prediction analysis which reported a potential alteration of splicing activities. As expected, the evaluation of the LD between rs2277065 and rs2277066 showed a $D^{\prime}=0.94$ thereby showing a high LD. Given these results, we extended the LD analysis to the whole set of SNPs considered in this work (rs2227282, rs2285700, rs10062446, rs2897442, rs2277066 and rs2277065). The analysis reported a $\mathrm{D}^{\prime}=0.79$ in cases, while the control group showed a $\mathrm{D}^{\prime}=0.18$. On the basis of our results, we performed the haplotype analysis considering the SNPs of interest and obtaining several haplotype combinations. As shown in Table 5, 2 haplotypes resulted to be significantly associated with PsA, particularly: C-A-A-A-G-A reported a $p=2.92^{*} 10^{-7}$ and $\mathrm{OR}=1.98(95 \% \mathrm{CI}: 1.52-2.59)$ while G-GT-G-G-A had a $p=3.8^{*} 10^{-3}$ and an OR=0.56 (95\%CI: 0.410.75 ). These data suggested that the PsA susceptibility region is not localized within the promoter region; rather

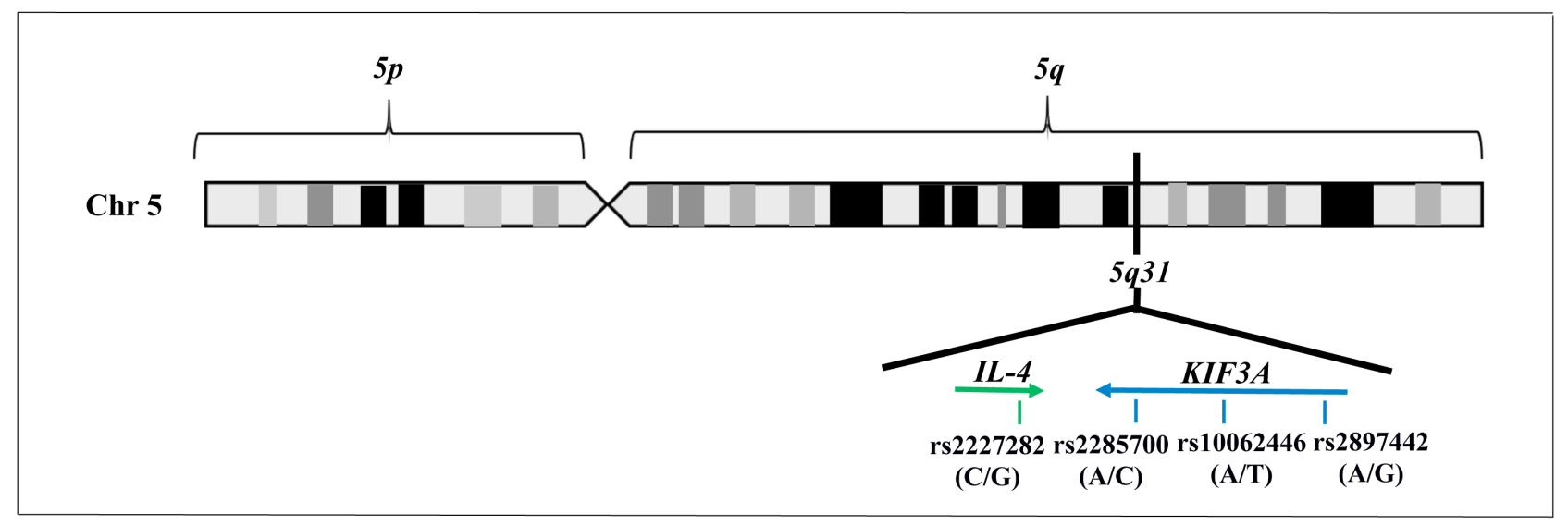

Figure 1: Illustration of $5 q 31$ locus, including the genes and the SNPs selected by in-silico analysis. 
Table 2: Case-control study performed on PsA and PsV patients

\begin{tabular}{|c|c|c|c|c|c|c|}
\hline Disease & Locus & SNPs & $\begin{array}{c}\text { Alleles } \\
\text { Frequencies } \\
(\mathbf{C} / \mathbf{C n})\end{array}$ & $p$ & OR (95\% CI) & Allele Effect \\
\hline \multirow{8}{*}{ PsA } & $5 q 31$ & rs2227282 & $\mathrm{C}: 0.79 / 0.72$ & $2.09601^{*} 10^{-}$ & $1.4(1.12-1.73)$ & Risk \\
\hline & & & G:0.21/0.28 & 5 & $0.7(0.57-0.89)$ & Protective \\
\hline & $5 q 31$ & rs2285700 & $\mathrm{A}: 0.79 / 0.75$ & $3^{*} 10^{-3}$ & $1.3(0.51-1.60)$ & Risk \\
\hline & & & $\mathrm{C}: 0.21 / 0.25$ & & $0.8(0.62-0.98)$ & Protective \\
\hline & $5 q 31$ & rs10062446 & $\mathrm{A}: 0.79 / 0.75$ & $5^{*} 10^{-3}$ & $1.3(1.0-1.57)$ & Risk \\
\hline & & & $\mathrm{T}: 0.21 / 0.25$ & & $0.8(0.67-0.93)$ & Protective \\
\hline & $5 q 31$ & rs2897442 & $\mathrm{A}: 0.77 / 0.72$ & $1.2^{*} 10^{-4}$ & $1.4(1.09-1.71)$ & Risk \\
\hline & & & $\mathrm{G}: 0.23 / 0.28$ & & $0.7(0.59-0.92)$ & Protective \\
\hline \multirow{8}{*}{ PsV } & $5 q 31$ & rs2227282 & $C: 0.69 / 0.72$ & ns & - & - \\
\hline & & & $\mathrm{G}: 0.31 / 0.28$ & & - & - \\
\hline & $5 q 31$ & rs2285700 & $\mathrm{A}: 0.72 / 0.75$ & ns & - & - \\
\hline & & & $C: 0.28 / 0.25$ & & - & - \\
\hline & $5 q 31$ & rs 10062446 & $\mathrm{~A}: 0.72 / 0.75$ & ns & - & - \\
\hline & & & $\mathrm{T}: 0.28 / 0.25$ & & & \\
\hline & $5 q 31$ & rs2897442 & $\mathrm{A}: 0.74 / 0.72$ & ns & - & - \\
\hline & & & G:0.26/0.28 & & - & - \\
\hline
\end{tabular}

Table 3: Haplotype analysis on $I L-4 / K I F 3 A$ block

\begin{tabular}{lccccc}
\hline Haplotypes & $\begin{array}{c}\text { PsA patients } \\
\text { Frequencies }\end{array}$ & $\begin{array}{c}\text { Control subjects } \\
\text { Frequencies }\end{array}$ & $\boldsymbol{p}$ & OR (95\% CI) & Haplotype Effect \\
\hline C-A-A-A & 0.78 & 0.68 & $1.70652^{*} 10^{-6}$ & $1.6(1.2-2.0)$ & Risk \\
G-A-A-A & 0.02 & 0.01 & $\mathrm{~ns}$ & - & - \\
G-C-T-A & 0.001 & 0.001 & $\mathrm{~ns}$ & - & - \\
C-C-A-G & 0.001 & - & $\mathrm{ns}$ & - & - \\
G-C-G-A & 0.001 & - & $\mathrm{ns}$ & - & - \\
C-C-T-G & 0.013 & 0.01 & $\mathrm{~ns}$ & - & - \\
G-A-A-G & 0.008 & 0.01 & $\mathrm{~ns}$ & - & - \\
G-A-T-G & 0.006 & - & $\mathrm{ns}$ & - & - \\
G-C-T-G & 0.17 & 0.29 & $1.70652^{*} 10^{-6}$ & $0.6(0.5-0.8)$ & Protective \\
\hline
\end{tabular}

Only the haplotypes with a frequency $>2 \%$ are reported.

it can be specifically restricted to the $I L 4 / K I F 3 A$ block. In particular, while the haplotype C-A-A-A-G-A (composed of risk alleles at $I L 4 / K I F 3 A$ block and $K I F 3 A$ promoter region) reported significant association values showing a risk effect on PsA susceptibility, the haplotype G-G-T-G$\mathrm{G}-\mathrm{A}$ (composed of protective alleles at $I L 4 / K I F 3 A$ block and risk alleles at the $K I F 3 A$ promoter region) showed a protective effect (Table 5).

\section{Immunohistochemical studies}

Successively, we decided to assess the tissue localization of KIF3A through immunohistochemistry, in order to observe a differential expression of KIF3A in patients compared to control subjects. The immunohistochemical studies documented that KIF3A immunoreactivity was faint in tissue biopsies of normal 
Table 4: Case-control study performed on $K I F 3 A$ promoter region in PsA patients

\begin{tabular}{|c|c|c|c|c|c|c|}
\hline Disease & Locus & SNPs & Alleles & $p$ & OR $(95 \%$ CI $)$ & Allele Effect \\
\hline \multirow{4}{*}{ PsA } & $5 q 31$ & rs2277065 & A & 0.001 & $\begin{array}{c}1.70(1.22- \\
2.36)\end{array}$ & Risk \\
\hline & \multirow{3}{*}{$5 q 31$} & \multirow{3}{*}{ rs2277066 } & G & \multirow{3}{*}{0.004} & $\begin{array}{c}0.59(0.42- \\
0.81)\end{array}$ & Protective \\
\hline & & & G & & $\begin{array}{c}1.52(1.14- \\
2.04)\end{array}$ & Risk \\
\hline & & & $\mathrm{C}$ & & $\begin{array}{c}0.65(0.49- \\
0.88)\end{array}$ & Protective \\
\hline
\end{tabular}

Table 5: Haplotype analysis performed on $I L-4 / K I F 3 A$ block and $K I F 3 A$ promoter region

\begin{tabular}{lccccc}
\hline Haplotypes & $\begin{array}{c}\text { PsA patients } \\
\text { Frequencies }\end{array}$ & $\begin{array}{c}\text { Control subjects } \\
\text { Frequencies }\end{array}$ & $\boldsymbol{p}$ & OR (95\% CI) & Haplotype Effect \\
\hline C-A-A-A-G-A & 0.76 & 0.63 & $2.92^{*} 10^{-7}$ & $1.98(1.52-2.59)$ & Risk \\
G-C-T-G-G-A & 0.13 & 0.21 & $3.8^{*} 10^{-3}$ & $0.56(0.41-0.75)$ & Protective \\
G-C-T-G-C-G & 0.04 & 0.03 & ns & - & - \\
\hline
\end{tabular}

The table shows only the haplotypes with a frequency $>2 \%$. Risk alleles are written in bold characters.

synovial tissue $(<25 \%$ positive cells on average, Figure 2a-2b), whereas KIF3A expression appeared more marked and present in more than $80 \%$ of positive cells on average in rheumatoid synovial tissue (Figure 2c2d). Moreover, KIF3A expression was almost faint in macroscopically normal hip cartilage with less than $25 \%$ of positive cells on average (Figure 2e-2f), while KIF3A expression markedly increased ( $>80 \%$ positive cells on average; Figure $2 \mathrm{~g}-2 \mathrm{~h}$ ) in hypertrophic chondrocytes from osteoarthritic cartilage. Semiquantitative evaluation confirmed the higher KIF3A expression in pathological compared to normal tissues $\left({ }^{* *} p<0,007\right.$ and ${ }^{*} p<0,013$, Figure 3). Altogether, the presented data highlights the potential involvement of a differential expression and function of particular bone-related genes (such as KIF3A and $I L-4)$ in the etiopathogenesis of PsA.

\section{DISCUSSION}

Among the connective tissues, the bone has one of the most dynamic metabolisms with constant turnover and remodelling activities. The homoeostasis between these two activities is maintained by the proper functioning of the osteoblasts (osteogenesis) and osteoclasts (bone resorption). In physiological conditions, bone homoeostasis is perfectly balanced and can promptly respond to different kinds of internal/ external stimuli (mechanical stress, inflammation, trauma, bone regeneration). In the presence of PsA, the bone homoeostasis is heavily disrupted leading to bone erosion, structural alteration and new bone formation [21]. Moreover, patients with PsA often experience a $\mathrm{BMD}$ reduction that is responsible for the bone loss and increased predisposition to fractures [21-23]. However, the contribution of bone metabolism genes on the onset of PsA still remains unknown.

The results concerning the study of the $5 \mathrm{q} 31$ genomic regions falling within 132,692,628-132,737,638 bp in our cohort provided interesting insights into the potential involvement of bone-related genes in PsA etiopathogenesis. In fact, the strong genetic association of $K I F 3 A$ and $I L-4$ identified them as two novel susceptibility genes for PsA. Moreover, it is a matter of fact that the $5 \mathrm{q} 31$ locus, including both the genes, is known to be characterized by a complex LD pattern. As expected, the LD study of rs2227282, rs2285700, rs 10062446 and rs2897442 in our PsA cohort reported a $D^{\prime}=0.95$. This result indicates the presence of a specific block in high LD within 132,692,628-132,737,638 bp of 5q31, representing additional evidence of association of the $5 \mathrm{q} 31$ region in PsA patients. In fact, our previous study on 1962 PsA patients revealed association of $\mathrm{rs} 715285$ (A/G; located between CSF2 and P4HA2 genes, 132,149,690 bp) [14]. LD analysis (data not shown) confirmed the independence of the rs 715285 signal from the $I L 4 / K I F 3 A$ block (rs2227282, rs2285700, rs10062446 and rs2897442). Moreover, the analysis of the allele architecture revealed two associated-haplotypes with the same association degree $\left(p=1.70652^{*} 10^{-6}\right)$ but opposite effect on the susceptibility to PsA. In particular, the haplotype C-A-A-A was recognized 


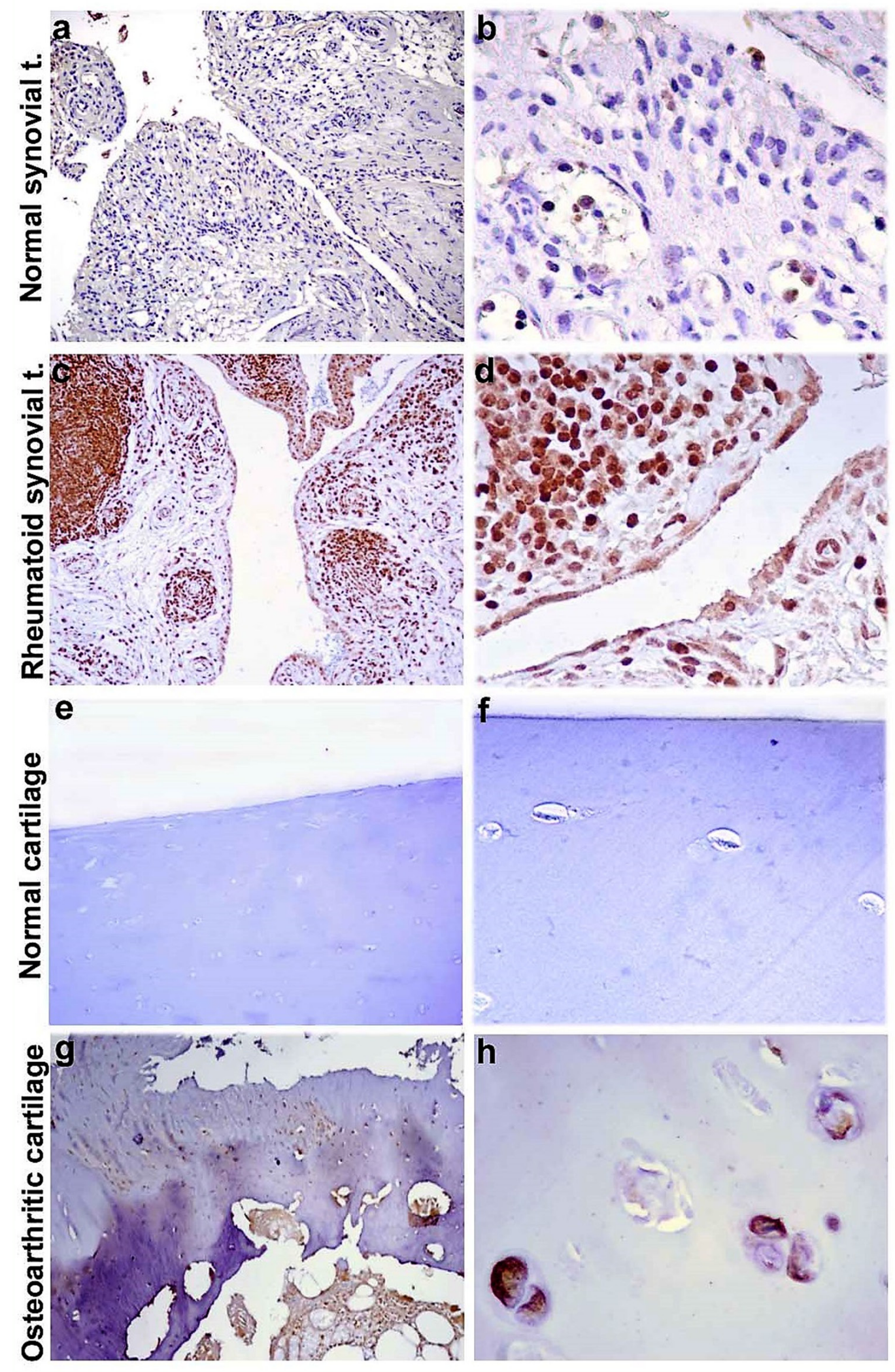

Figure 2: KIF3A immunostaining of synovial and cartilaginous tissue. Representative images of KIF3A immunostaining of osteoarthritic and rheumautoid synovial tissue (a-d) and macroscopically normal and osteoarthritic cartilage (e-h). Diaminobenzidine as chromogen; original magnification: $\mathrm{a}, \mathrm{c}$, e and $\mathrm{g}$ at 100X; b, d, f and h at 400X. Abbreviations: normal synovial t.= normal synovial tissue; rheumatoid synovial $t_{\text {. }}=$ rheumatoid synovial tissue . 
to increase the risk for disease $(\mathrm{OR}=1.6)$ and was found more frequently in our patient cohort. On the other hand, the haplotype G-C-T-G was classified as protective for PsA $(\mathrm{OR}=0.6)$ and showed a higher frequency in our control subjects. Interestingly, both haplotypes cover the $97 \%$ of variability of the $5 \mathrm{q} 31$ region of interest. Successively, we extended our research to the $K I F 3 A$ promoter region in order to detect variants that may influence or modify the transcriptional activity of the gene. On this subject, the rs2277065 and rs2277066 SNPs ended up to be associated with a higher susceptibility to PsA, as demonstrated by the significant association values $(p=0.001$ and $\mathrm{OR}=1.70$; $p=0.004$ and $\mathrm{OR}=1.52$, respectively). The association of both the SNPs with the disease was independent from the rs715285, as shown by the low LD among those polymorphisms $\left(D^{\prime}=0.10\right)$. Taken together, our results support the thesis that $5 q 31$ is a locus specific for PsA. On this subject, it is important to remark that $K I F 3 A$ and $I L-4$ gave non-significant data from the genotyping/biostatistic analysis performed on our PsV cohort. The divergence of results between PsA and PsV samples may be linked to the molecular function displayed by the KIF3A and IL-4 proteins. In fact, both are known to take part in the modulation of osteogenic and remodelling activities of the bone at different levels. Several studies have demonstrated the role of IL-4 in the regulation of the osteoclastogenesis in vitro and in vivo. In particular, IL-4 showed to be able to prevent the differentiation of both the precursors and the mature osteoclasts by suppressing the expression of several modulators, such as RANKL, STAT6, NF-Kb and calcium signalling [24-26]. Concerning the role of KIF3A in the bone metabolism, recent research revealed that it participates in the commitment of osteoblast differentiation through a primary cilia induced-mechanism. This pathway was found to be activated in response to physical and mechanical stresses of the bone [27-29]. The mechanical loading plays a crucial role in bone remodelling and depends on external stimuli, such as ageing, weight, stress and trauma. In particular, mechanical stress and trauma have been supposed to be triggers of joint inflammation (especially enthesitis) and new bone formation in PsA and spondyloarthritis.

In fact, the lack of mechanical loading increases the turnover toward a more prevalent resorption activity, while the excessive mechanical loading damages the bone tissue and induces the remodelling [29-30]. The alteration of bone homoeostasis by excessive mechanical loading, damage and uncontrolled remodelling activity may thus contribute to the onset of arthropaties (osteoporosis, osteoarthritis, PsA) [21, 29-30]. Our results from the immunohistochemical studies clearly showed a marked and significant difference of KIF3A expression in rheumatoid and osteoarthritic tissue compared to normal synovial and cartilage tissues thus supporting the potential involvement of KIF3A in the etiopathogenesis of arthropaties. Unfortunately, any sinovial or

\section{synovial tissue}

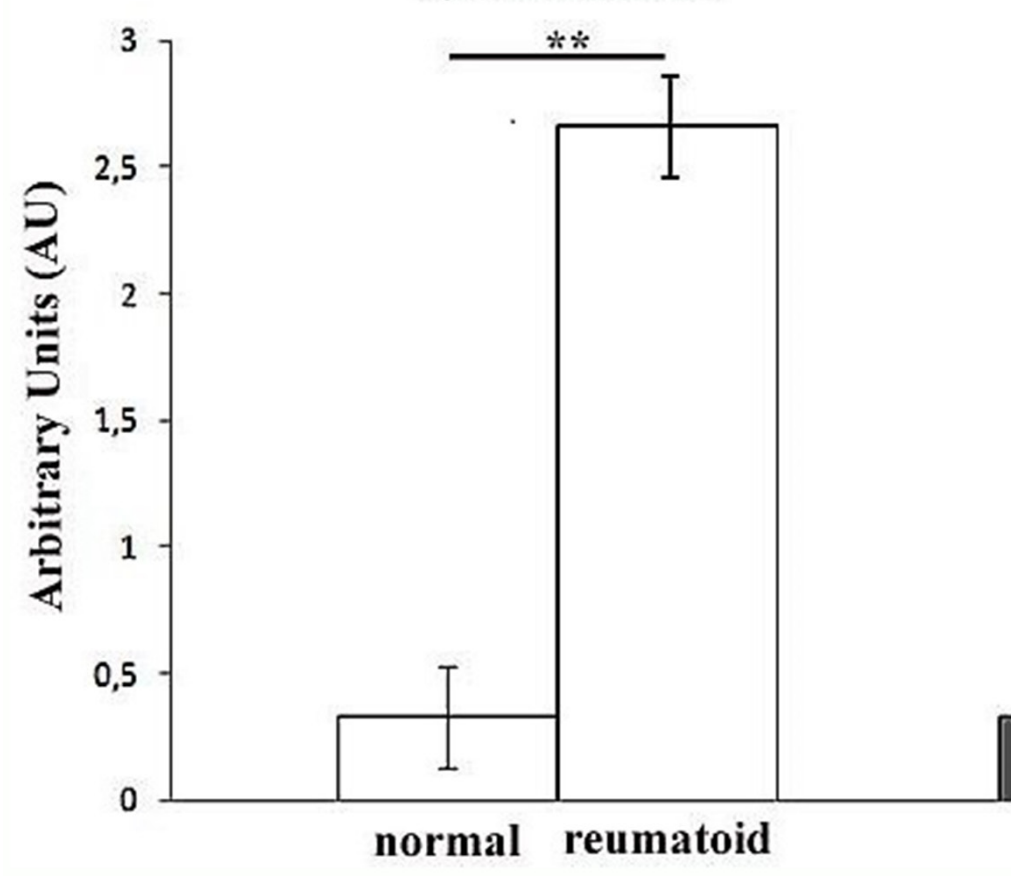

\section{hip cartilage}

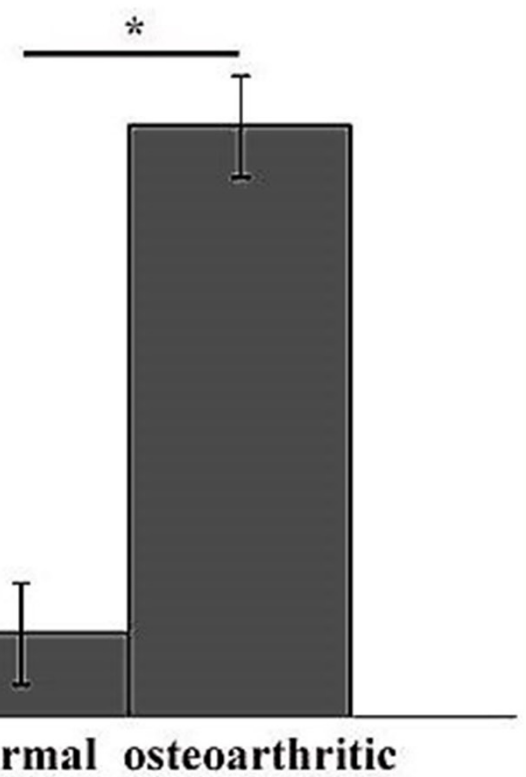

Figure 3: Semiquantitative evaluation of KIF3A immunostaining. Bar graph showing the higher levels of KIF3A immunostainings in rheumatoid compared to normal synovial tissue $\left({ }^{* *} \mathrm{p}<0,007\right)$ and in osteoarthritic compared to normal hip cartilage $\left({ }^{*} \mathrm{p}<0,013\right)$. Results are expressed as mean values \pm SEM. 
cartilage tissue of patients with PsA were available for immunohistochemistry studies. These kind of samples are really difficult to find, although this will be certainly collected and analysed in the next future. Moreover, other studies are needed to investigate those KIF3A-dependent pathways which influence the maintenance of skin homoeostasis and skeletal morphogenesis. On the basis of these results, it would have been interesting to study the expression profile of the KIF3A gene in PsA patients with respect to control subjects. The gene is known to be highly expressed in the synovial tissue and, at very low levels, in the whole blood. Unfortunately, the expression analysis was not possible due to the lack of fresh synovial tissues from patients with PsA. It is important to remind that the collection procedure is highly invasive and painful for the patients and often disagree to donate a synovial tissue sample.

To date, the knowledge of PsA has been mainly concentrated on the research of genomic biomarkers and "molecular effectors" participating in immunologic, inflammatory and epidermal differentiation pathways leading to the onset and progression of disease. However, the role of bone metabolism and remodelling activities in PsA remains poorly understood. Few studies have tried to define which are the molecular players contributing to the disruption of bone homeostasis in PsA. The genomic features potentially linked with this process seem to be unexplored, since none of the genes controlling bone metabolism have been associated with the disease. In this context, we pointed our attention on $K I F 3 \mathrm{~A}$ gene and its flanking regions $(132,692,628-132,737,638 \mathrm{bp})$ considering its positional and functional properties potentially implicated in PsA. Our work revealed that $K I F 3 A$ and $I L-4$ are associated with PsA, indicating a clear implication of bone metabolism genes. These results pave the way for the characterization of a cluster of genes within the PsA-associated 5q31 locus in which immune/inflammatory and bone metabolism genes may actually interact in a unique etiopathogenetic pathway. In conclusion, the present data should be regarded as a starting point for future studies aimed to enrich the knowledge concerning 5q31 locus and its specific association with PsA, as well as the contribution of bonerelated genes to the disease.

\section{MATERIALS AND METHODS}

\section{Ethics statement}

Investigation has been conducted in accordance with the ethical standards and according to the Declaration of Helsinki and according to national and international guidelines and has been approved by the authors' institutional review board.

\section{Materials and methods}

The main objective of the present study was the investigation of $K I F 3 A$ gene and its flanking regions, in order to find evidence of potential connection between bone-related genes and PsA.

This study was designed as a case-control study involving a cohort of 1526 subjects recruited from "Tor Vergata" General Hospital in Rome. The cohort was subdivided in 500 patients affected with PsA, 426 patients affected with PsV and 600 healthy controls. The diagnosis of PsV and PsA were fulfilled according to the classical clinical criteria and the CASPAR Study Group criteria [31]. All patients were negative for the rheumatoid factor. Concerning the control subjects, they did not report evidence for PsV or PsA at the time of recruitment. Patients and control subjects have been recruited upon signature of informed consent. A blood sample was taken from all of the subjects in order to extract the genomic DNA. The DNA extraction was performed by the EZ1 Advanced XL automated extractor and the EZ1 DNA Blood $200 \mu \mathrm{l}$ Kit (Qiagen).

The genomic variability of $5 \mathrm{q} 31$ locus was evaluated with special attention to the $K I F 3 A$ gene and its flanking regions $(132,692,628-132,737,638$ bp). To this purpose, we first selected the SNPs that were able to represent the maximum variability of the region through the online tool "HapMap3 Genome Browser release \#2 (Phase 3-genotypes, frequencies \& LD)". Afterwards, we chose the fewest number of SNPs able to characterize the maximum genomic variability of the 132,692,628$132,737,638$ bp region (GRCh38). The SNPs were genotyped in all of the 1526 subjects in order to evaluate their association with PsA and PsV. The genotyping analysis was performed by TaqMan assays on a 7500 Fast Real Time PCR device (Applied Biosystems). Each Real Time PCR run was performed using a negative control and three positive control samples (homozygote wild-type, homozygote variant, heterozygote) which were previously confirmed by direct sequencing. The PCR sequence reactions were performed with BigDye Terminator v3.1 (Applied Biosystems) according to the manufacturer's instructions. After purification with BigDyeXTerminator (Applied Biosystems), samples were run on ABI3130x1 (Applied Biosystems). The sequences were read by the Sequencing Analysis software v5.2 (Applied Biosystems).

The genotyping data obtained by Real Time PCR were interpreted using the Sequence Detection System 2.1 software (Applied Biosystems). In addition, the promoter region of $K I F 3 A$ was initially resequenced in a small cohort of patients $(n=50)$ in order to search for any variation that may alter the transcriptional profile of the gene in patients with PsA. Successively, the study of the $K I F 3 A$ promoter region was extended to the whole study 
cohort through the genotyping analysis by TaqMan assays and Real Time PCR as previously described.

Concerning the evaluation of KIF3A expression on synovial and cartilage tissues, the traditional immunohistochemistry approach was adopted. Fourmicrometer-thick serial sections were obtained from anonymised formalin-fixed, paraffin-embedded tissue samples $(\mathrm{n}=12)$ from Paraffin Block Archive of Anatomic Pathology of "Tor Vergata" University. Tissue samples included normal synovial tissue, normal hip, rheumatoid synovial tissue and osteoarthritic cartilage. Anonymised sections were stained with haematoxylin and eosin [32] for microscopic examination or employed for immunohistochemistry. For the latter, after deparaffinization and blocking of endogenous peroxidase activity with $0.2 \% \mathrm{H}_{2} \mathrm{O}_{2}$ and heat-mediated antigen retrieval, sections were incubated with polyclonal rabbit anti-KIF3A (1:200, ABIN1387574, antibodiesonline Inc.), followed by biotin-labelled goat antirabbit secondary antibody and streptavidin-horseradish peroxidase conjugated (1:100); 3,3-diaminobenzidine as final chromogen and haematoxylin as counterstaining were used [33]. All immunohistochemical procedures included internal positive and negative controls. KIF3A positivity was quantified using an arbitrary semiquantitative grading of the percentage of positive cells $(0=0 \% ; 1=0-25 \%$; $2=26-50 \% ; 3=51-100 \%$ ) from two researchers, with an intervariability $<5 \%$ [34].

Genotyping results were subjected to biostatistical analysis in order to evaluate the association with PsV and PsA. First, the Hardy-Weinberg equilibrium was confirmed in each group of the cohort (PsA, PsV and control subjects). Afterwards, the association of the genotyped SNPs were measured by calculating the $p$-value (p) through a $2 \times 2$ contingency tables (http://www.physics. csbsju.edu/stats/contingency_NROW_NCOLUMN form.html). The statistical associations were considered significant for $p<0.05$ with a $95 \%$ confidence interval. The strength of association was established by calculating the Odd Ratio (OR, http://www.hutchon.net/ConfidOR.htm).

Immunohistochemical data were analysed using Student's $t$-test. The results of semiquantitative evaluation were represented as the mean \pm SEM. In general, $p<0.05$ was considered statistically significant. SPSS v16.0 (SPSS Incorporated) was used for statistical analyses.

The haplotype analysis was performed on the software UNPHASED version 3.1.7 (5/6/13) [35] and replicated on Haploview [36]. The evaluation of Linkage Disequilibrium for the SNPs of interest was assessed by the dedicated online tool (http://pharmgat.org/Tools/ pbtoldplotform) and Haploview. Furthermore, the genotyping results were analysed by bioinformatic tools in order to characterize the SNPs (Single Nucleotide Polymorphisms) and the molecular function of the genes included in the region of interest (NCBI, HapMap, Ensembl, Genome Browser, GeneCards, 1000 Genome
Browser). The polymorphisms were subjected to prediction analysis with the purpose of assessing any potential splice site variation (Mutation Taster and Human Splice Finder).

\section{Abbreviations}

PsA: Psoriatic Arthritis; PsV: Psoriasis Vulgaris; KIF3A: Kinesin Family Member 3A; $p$ : p-value; OR: Odd Ratio; LD: Linkage Disequilibrium; SNPs: Single Nucleotide Polymorphisms; BMD: Bone Mineral Density.

\section{Author contributions}

$\mathrm{AO}, \mathrm{EG}$, made substantial contributions to conception and design of the study. RC, CS, MR, LM, GC made substantial contributions to acquisition of data, analysis and interpretation of data. RC, CS, GC, AO, EG have been involved in drafting the manuscript. JB, UH, $\mathrm{AR}, \mathrm{AB}$ revising it critically for important intellectual content. RC, CS, MR, LM, GC, JB, UH, SP, FS, AR, AB, $\mathrm{GN}, \mathrm{AO}, \mathrm{EG}$ have given final approval of the version to be published. SP, FS, AR, AB, GN, AO, EG are agreed to be accountable for all aspects of the work in ensuring that questions related to the accuracy or integrity of any part of the work are appropriately investigated and resolved.

\section{CONFLICTS OF INTEREST}

The authors declare that they have no competing interests.

\section{FUNDING}

Not applicable.

\section{REFERENCES}

1. O'Rielly DD, Rahman P. Genetic, epigenetic and pharmacogenetic aspects of psoriasis and psoriatic arthritis. Rheum Dis Clin North Am. 2015; 41:623-642.

2. Rahman P, Elder JT. Genetics of psoriasis and psoriatic arthritis: a report from the GRAPPA 2010 annual meeting. J Rheumatol. 2012; 39:431-433.

3. O'Rielly DD, Rahman P. Genetics of susceptibility and treatment response in psoriatic arthritis. Nat Rev Rheumatol. 2011; 7:718-732.

4. Haroon M, FitzGerald O. Psoriatic arthritis: complexities, comorbidities and implications for the clinic. Expert Rev Clin Immunol. 2016; 28:1-12.

5. Gelfand JM, Gladman DD, Mease PJ, Smith N, Margolis DJ, Nijsten T, Stern RS, Feldman SR, Rolstad T. Epidemiology of psoriatic arthritis in the population of the United States. J Am Acad Dermatol. 2005; 53:573. 
6. Rahman P, Elder JT. Genetic epidemiology of psoriasis and psoriatic arthritis. Ann Rheum Dis. 2005; 64:37-39.

7. Cascella R, Strafella C, Longo G, Maccarone M, Borgiani P, Sangiuolo F, Novelli G, Giardina E. Pharmacogenomics of multifactorial diseases: a focus on psoriatic arthritis. Pharmacogenomics. 2016; 17:943-951.

8. O'Rielly DD, Rahman P. Genetics of psoriatic arthritis. Best Pract Res Clin Rheumatol. 2014; 28:673-685.

9. Giardina E, Hüffmeier U, Ravindran J, Behrens F, Lepre T, McHugh NJ, Korendowych E, Burkhardt H, Novelli G, Reis A. Tumor necrosis factor promoter polymorphism TNF* 857 is a risk allele for psoriatic arthritis independent of the PSORS1 locus. Arthritis Rheum. 2011; 63:3801-3806.

10. Hüffmeier U, Uebe S, Ekici AB, Bowes J, Giardina E, Korendowych E, Juneblad K, Apel M, McManus R, Ho P, Bruce IN, Ryan AW, Behrens F, et al. Common variants at TRAF3IP2 are associated with susceptibility to psoriatic arthritis and psoriasis. Nat Genet. 2010; 42:996-999.

11. Docampo E, Giardina E, Riveira-Muñoz E, De Cid R, Escaramís G, Perricone C, Fernández-Sueiro JL, Maymó J, González-Gay MA, Blanco FJ, Hüffmeier U, Lisbona MP, Martín J, et al. Deletion of LCE3C and LCE3B is a susceptibility factor for psoriatic arthritis: a study in Spanish and Italian populations and meta-analysis. Arthritis Rheum. 2011; 63:1860-1865.

12. Apel M, Uebe S, Bowes J, Giardina E, Korendowych E, Juneblad K, Pasutto F, Ekici AB, McManus R, Ho P, Bruce IN, Ryan AW, Behrens F, et al. Variants in RUNX3 contribute to susceptibility to psoriatic arthritis, exhibiting further common ground with ankylosing spondylitis. Arthritis Rheum. 2013; 65:1224-1231.

13. Tsoi LC, Spain SL, Knight J, Ellinghaus E, Stuart PE, Capon F, Ding J, Li Y, Tejasvi T, Gudjonsson JE, Kang HM, Allen MH, McManus R, et al. Identification of 15 new psoriasis susceptibility loci highlights the role of innate immunity. Nat Genet. 2012; 44:1341-1348.

14. Bowes J, Budu-Aggrey A, Huffmeier U, Uebe S, Steel K, Hebert HL, Wallace C, Massey J, Bruce IN, Bluett J, Feletar M, Morgan AW, Marzo-Ortega H, et al. Dense genotyping of immune-related susceptibility loci reveals new insights into the genetics of psoriatic arthritis. Nat Commun. 2015; 6:7741.

15. Bowes J, Loehr S, Budu-Aggrey A, Uebe S, Bruce IN, Feletar M, Marzo-Ortega H, Helliwell P, Ryan AW, Kane D, Korendowych E, Alenius GM, Giardina E, et al. PTPN22 is associated with susceptibility to psoriatic arthritis but not psoriasis: evidence for a further PsA-specific risk locus. Ann Rheum Dis. 2015; 74:1882-1885.

16. McInnes IB. Psoriatic arthritis: embracing pathogenetic and clinical heterogeneity? Clin Exp Rheumatol. 2016; 34:9-11.

17. Lepre T, Cascella R, Ragazzo M, Galli E, Novelli G, Giardina E. Association of KIF3A, but not OVOL1 and ACTL9, with atopic eczema in Italian patients. Br J Dermatol. 2013; 168:1106-1108.
18. Scholey JM. Kinesin-2:a family of heterotrimeric and homodimeric motors with diverse intracellular transport functions. Annu Rev Cell Dev Biol. 2013; 29:443-469.

19. Qiu N, Xiao Z, Cao L, Buechel MM, David V, Roan E, Quarles LD. Disruption of Kif3a in osteoblasts results in defective bone formation and osteopenia. J Cell Sci. 2012; 125:1945-57.

20. Kolpakova-Hart E, Jinnin M, Hou B, Fukai N, Olsen BR. Kinesin-2 controls development and patterning of the vertebrate skeleton by Hedgehog- and Gli3-dependent mechanisms. Dev Biol. 2007; 309:273-284.

21. Paine A, Ritchlin C. Bone remodeling in psoriasis and psoriatic arthritis: an update. Curr Opin Rheumatol. 2016; 28:66-75.

22. Bertoldi I, Filippou G, Scirè CA, Picerno V, di Sabatino V, Adinolfi A, Pierguidi S, Galeazzi M, Frediani B. Disease Activity and Bone Mineral Density of MCP Joints in Patients with Rheumatoid and Psoriatic Arthritis: Is There a Correlation?-A Study in Patients Treated with Methotrexate and an Anti-TNF $\alpha$ Agent. ISRN Rheumatol. 2013; 2013:708323.

23. Borman P, Babaoğlu S, Gur G, Bingol S, Bodur H. Bone mineral density and bone turnover in patients with psoriatic arthritis. Clin Rheumatol. 2008; 27:443-447.

24. Mangashetti LS, Khapli SM, Wani MR. IL-4 inhibits bone-resorbing activity of mature osteoclasts by affecting NF-kappa B and Ca2+ signaling. J Immunol. 2005; 175:917-925.

25. Moreno JL, Kaczmarek M, Keegan AD, Tondravi M. IL-4 suppresses osteoclast development and mature osteoclast function by a STAT6-dependent mechanism: irreversible inhibition of the differentiation program activated by RANKL. Blood. 2003; 102:1078-1086.

26. Bendixen AC, Shevde NK, Dienger KM, Willson TM, Funk CD, Pike JW. IL-4 inhibits osteoclast formation through a direct action on osteoclast precursors via peroxisome proliferator-activated receptor $\gamma 1$. Proc Natl Acad Sci U S A. 2001; 98:2443-2448.

27. Leucht P, Monica SD, Temiyasathit S, Lenton K, Manu A, Longaker MT, Jacobs CR, Spilker RL, Guo H, Brunski JB, Helms JA. Primary cilia act as mechanosensors during bone healing around an implant. Med Eng Phys. 2013; 35:392-402.

28. Temiyasathit S, Tang WJ, Leucht P, Anderson CT, Monica SD, Castillo AB, Helms JA, Stearns T, Jacobs CR. Mechanosensing by the primary cilium: deletion of Kif3A reduces bone formation due to loading. PLoS ONE. 2012. https://doi.org/10.1371/0033368.

29. Chen JC, Hoey DA, Chua M, Bellon R, Jacobs CR. Mechanical signals promote osteogenic fate through a primary cilia-mediated mechanism. FASEB J. 2015; 30:1504-1511.

30. Barnas JL, Ritchlin CT. Etiology and Pathogenesis of Psoriatic Arthritis. Rheum Dis Clin North Am. 2015; 41:643-663. 
31. Taylor W, Gladman D, Helliwell P, Marchesoni A, Mease P, Mielants H, CASPAR Study Group. Classification criteria for psoriatic arthritis: development of new criteria from a large international study. Arthritis Rheum. 2006; 54:2665-2673.

32. Spagnoli LG, Orlandi A, Marino B, Mauriello A, De Angelis C, Ramacci MT. Propionyl-L-carnitine prevents the progression of atherosclerotic lesions in aged hyperlipemic rabbits. Atherosclerosis. 1995; 114:29-44.

33. Cervelli V, Scioli MG, Gentile P, Doldo E, Bonanno E, Spagnoli LG, Orlandi A. Platelet-rich plasma greatly potentiates insulin-induced adipogenic differentiation of human adipose-derived stem cells through a serine/ threonine kinase Akt-dependent mechanism and promotes clinical fat graft maintenance. Stem Cells Transl Med. 2012; 1:206-220.

34. Orlandi A, Francesconi A, Marcellini M, Ferlosio A, Spagnoli LG. Role of ageing and coronary atherosclerosis in the development of cardiac fibrosis in the rabbit. Cardiovasc Res. 2004; 64:544-552.

35. Dudbridge F. Likelihood-based association analysis for nuclear families and unrelated subjects with missing genotype data. Hum Hered. 2008; 66:87-98.

36. Barrett JC, Fry B, Maller J, Daly MJ. Haploview: analysis and visualization of LD and haplotype maps. Bioinformatics. 2005; 21:263-265. 